\title{
The Separable Complementation Property and Mrówka Compacta
}

\author{
Jesús Ferrer ${ }^{1}$ \\ ${ }^{1}$ Departamento de Anàlisis Matemático, Universidad de Valencia, Dr. Moliner, 50, 46100 Burjasot (Valencia), Spain \\ Correspondence: Jesús Ferrer, Departamento de Anàlisis Matemático, Universidad de Valencia, Dr. Moliner, 50, 46100 \\ Burjasot (Valencia), Spain. E-mail: Jesus.Ferrer@uv.es
}

Received: March 21, 2017 Accepted: July 15, 2017 Online Published: August 21, 2017

doi:10.5539/jmr.v9n5p30

URL: https://doi.org/10.5539/jmr.v9n5p30

\begin{abstract}
We study the separable complementation property for $C\left(K_{\mathcal{A}}\right)$ spaces when $K_{\mathcal{A}}$ is the Mrówka compact associated to an almost disjoint family $\mathcal{A}$ of countable sets. In particular we prove that, if $\mathcal{A}$ is a generalized ladder system, then $C\left(K_{\mathcal{A}}\right)$ has the separable complementation property ( $S C P$ for short) if and only if it has the controlled version of this property. We also show that, when $\mathcal{A}$ is a maximal generalized ladder system, the space $C\left(K_{\mathcal{A}}\right)$ does not enjoy the $S C P$.
\end{abstract}

Keywords: Mrówka compacta, mad families, Separable Complementation Property

MSC Subject Classifications: 46B10, 46B26

\section{Introduction}

In previous papers, see (Ferrer, J., 2009; Ferrer, J. \& Wójtowicz, M., 2011; Ferrer, J., Koszmider, P. \& W. Kubiś, 2013; Ferrer, J., 2014; Ferrer, J., 2009), we studied the controlled version of the separable complementation property (CSCP, for short) for general Banach spaces and in particular for $C\left(K_{\mathcal{P}}\right)$ spaces when $K_{\mathcal{P}}$ is the Mrówka compact associated to an almost disjoint family $\mathcal{A}$ of countable subsets of a given set. After seeing that $K$ being monolithic, see (Arkhangel'skii, A. V., 1992), is a necessary condition in order that the space $C(K)$ enjoys the $C S C P$, we proved this condition to be sufficient when $K$ is a Mrówka compact and moreover we also showed that this condition suffices in general when $K$ is a scattered compact such that each of its points, except possibly the ones in the top layer, admit a countable neighborhood base. When $\mathcal{A}$ is a maximal almost disjoint family, i.e., a mad family, since there are countably infinite sets which have an uncountable closure, it follows that the Mrówka compact $K_{\mathcal{A}}$ is not monolithic and so $C\left(K_{\mathcal{A}}\right)$ cannot have the $C S C P$. However, we do not know wether $C\left(K_{\mathcal{A}}\right)$, for $\mathcal{A}$ mad, may have the $S C P$. In this paper we try to give an answer to this problem.

For $\mathcal{A}$ being a generalized ladder system, we prove that $C\left(K_{\mathcal{A}}\right)$ has the $S C P$ if and only if it has the $C S C P$, which equals saying that $K_{\mathcal{A}}$ must be monolithic. Consequently, we obtain that, for $\mathcal{A}$ a maximal generalized ladder system, the space $C\left(K_{\mathcal{A}}\right)$ does not have the $S C P$.

In the following, if $K$ is a compact topological space (always Hausdorff), by $C(K)$ we mean the Banach space formed by all real-valued continuous functions defined in $K$ provided with the sup norm. For $A$ being a subset of the compact $K$, by $C_{A}(K)$ we denote the closed subspace of $C(K)$ formed by the functions which vanish in each point of $A$.

\section{About Almost Disjoint Families}

For the sake of completeness, we shall give some auxiliary details concerning almost disjoint families, maximal almost disjoint families and their associated Mrówka compacta.

Let $S$ be an infinite set. A collection $\mathcal{A}$ of countably infinite subsets of $S$ is said to be almost disjoint whenever every two distinct members of $\mathcal{A}$ have finite intersection. We shall assume in the following that $\mathcal{A}$ is an infinite almost disjoint family. By Zorn's Lemma, it is easy to see that there exist almost disjoint families which are maximal respect to setinclusion, such families are called mad families.

Let $\psi(S, \mathcal{A})$ denote the space with underlying set $S \cup \mathcal{A}$ and with the topology having as a base all singletons $\{s\}$ for $s \in S$, and all sets of the form $\{A\} \cup B$ where $A \in \mathcal{A}$ and $B$ is a cofinite subset of $A$. For $S=\mathbf{N}$, the positive integers, and $\mathcal{A}$ a mad family in $\mathbf{N}$, the space $\psi(\mathbf{N}, \mathcal{A})$ was studied by Mrówka (Mrówka, S., 1977). Also, for $S$ uncountable and $\mathcal{A}$ mad, some properties of the space $\psi(S, \mathcal{A})$ are studied in (Dow, A. \& Vaughan, J. E., 2009).

It is simple to check that $\psi(S, \mathcal{A})$ is a Hausdorff first countable locally compact space such that $S$ is dense. By $K_{\mathcal{A}}$ we denote the one-point compactification of $\psi(S, \mathcal{A})$, i.e., $K_{\mathcal{A}}=\psi(S, \mathcal{A}) \cup\{\infty\}$, and it is known as a Mrówka compact. It is also straightforward to notice that $K_{\mathcal{A}}$ is a scattered compact of height 3 .

\footnotetext{
${ }^{1}$ The author has been partially supported by MINECO and FEDER Project MTM2014-57838-C2-2-P.
} 
Mrówka compacta, although apparently quite simple to understand, have turned out to be a class in which the properties of having the $S C P$, having the $C S C P$ or being weakly compactly generated, which for these $C(K)$ spaces is equivalent to be isomorphic to some $c_{0}(\Gamma)$, can be separated for the corresponding function spaces. In (Ferrer, J., Koszmider, P. \& W. Kubiś, 2013) an example of a Mrówka compact $K_{\mathcal{A}}$ is given such that $C\left(K_{\mathcal{A}}\right)$ has the $S C P$ but not the $C S C P$, and also in that reference a broad class of Mrówka compacta whose function space enjoys the CSCP but cannot be isomorphic to any $c_{0}(\Gamma)$ space is highlighted, namely the non-trivial ladder systems in $\omega_{1}$.

The following is a basic result on the structure of mad families. Given two subsets of $S$, by $A \subset^{*} B$ we mean that $A \backslash B$ is a finite set and we shall say that $A$ is almost contained in $B$.

Lemma 1. Let $\mathcal{A}$ be a mad family in $S$. Then, for each infinite sequence $\left\{A_{j}: j \geq 1\right\}$ of distinct members of $\mathcal{A}$, the closure in $K_{\mathcal{A}}$ of the set $\cup_{j \geq 1} A_{j}$ has uncountable cardinality.

Proof. Let $\left\{A_{j}: j \geq 1\right\}$ be an infinite sequence of distinct members of the mad family $\mathcal{A}$. Let $N:=\cup_{j \geq 1} A_{j}$. We consider the following subfamily of $\mathcal{A}$

$$
\mathcal{A}_{N}:=\{A \in \mathcal{A}: A \cap N \text { is an infinite set }\} .
$$

Then, it is easy to see that the closure of $N$ in $K_{\mathcal{A}}$, which we denote by $\bar{N}$, is

$$
\bar{N}=N \cup \mathcal{A}_{N} \cup\{\infty\} .
$$

Hence, it all reduces to show that the collection $\mathcal{A}_{N}$ is not countable. Assuming this is not so, let us suppose that $\mathcal{A}_{N}=\left\{B_{j}: j \geq 1\right\}$. It is obvious, since $\mathcal{A}_{N}$ contains the collection $\left\{A_{j}: j \geq 1\right\}$, that the sequence $\left\{B_{j}: j \geq 1\right\}$ has infinite terms. We construct inductively the set $C=\left\{s_{j}: j \geq 1\right\}$ such that, for each $j, s_{j} \in N \cap B_{j} \backslash B_{1} \backslash \ldots \backslash B_{j-1}$ :

For $j=1$, since $N \cap B_{1}$ has infinitely many elements, take $s_{1}$ to be any element of $N \cap B_{1}$. For $j=2$, since $N \cap B_{2}$ is infinite and $B_{2} \cap B_{1}$ is finite, take $s_{2} \in N \cap B_{2} \backslash B_{1}$. For $j=3$, since $N \cap B_{3}$ is infinite and $B_{3} \cap\left(B_{2} \cup B_{1}\right)$ is finite, take $s_{3} \in N \cap B_{3} \backslash B_{1} \backslash B_{2}$, and so on. Hence, we have that, for each $j \geq 2, s_{j} \in N \cap B_{j} \backslash B_{1} \backslash \ldots \backslash B_{j-1}$. Clearly, the set $C$ is a countably infinite subset of $S$. Besides, given an arbitrary member $A \in \mathcal{A}$, we consider two possibilities:

Case 1. $A \notin \mathcal{A}_{N}$. Then, this means that $A \cap N$ is finite and, since $C \subseteq N$, it follows that $A \cap C$ is also finite.

Case 2. $A \in \mathcal{A}_{N}$. Now, there is $j \geq 1$ such that $A=B_{j}$. Thus, the intersection $A \cap C=B_{j} \cap C$ is also finite, since it is contained in the set $\left\{s_{1}, s_{2}, \ldots, s_{j}\right\}$.

We have then shown that $\mathcal{A} \cup\{C\}$ is an almost disjoint family. Since $C \notin \mathcal{A}$, this contradicts the maximality of $\mathcal{A}$.

In the following we recall the notion of ladder system in $\omega_{1}$. Ladder systems were originally used by R. Pol, see (Pol, R., 1979), to give the first example of a weakly Lindelöf $C(K)$ space such that $K$ is not a Corson compact. We used ladder systems in (Ferrer, J., Koszmider, P. \& W. Kubiś, 2013) to prove that there are Mrówka compact spaces $K_{\mathcal{A}}$ which are monolithic, hence its associated function space $C\left(K_{\mathcal{A}}\right)$ has the $C S C P$, but $K_{\mathcal{A}}$ is not even a continuous image of a Valdivia compact and so $C\left(K_{\mathcal{A}}\right)$ is not isomorphic to any $c_{0}(\Gamma)$ space.

We shall start by stating what a ladder system in $\omega_{1}$ is: Given a set $L$ of countable limit ordinals, a ladder system indexed by $L$ is a family of the form

$$
\mathcal{A}_{L}=\left\{A_{\delta}: \delta \in L\right\},
$$

where, for each $\delta \in L, A_{\delta}=\left\{\alpha_{\delta j}: \quad j \geq 1\right\}$ is a strictly increasing sequence of ordinals such that $\sup _{j} \alpha_{\delta j}=\delta$. It is straightforward that $\mathcal{A}_{L}$ is always an almost disjoint family in $\omega_{1}$ such that its associated Mrówka compact $K_{\mathcal{A}_{L}}$ is monolithic. When the set $L$ is stationary, i.e., it intersects every order-closed unbounded (club) subset of $\omega_{1}$, see (Jech, T., 2003), then the ladder system $\mathcal{A}_{L}$ is said to be non-trivial and the Mrówka compact $K_{\mathcal{A}_{L}}$ is no continuous image of a Valdivia compact.

We now introduce the notion of generalized ladder system in $\omega_{1}$. For a set $A$ of countable ordinals, by $A^{(1)}$ we denote the set of all order-accumulation points of $A$. An almost disjoint family $\mathcal{A}$ in the set $\omega_{1}$ is said to be a generalized ladder system whenever, for each $A \in \mathcal{A}$, we have that $A^{(1)}=\{\sup (A)\}$. Notice that, although every ladder system is a generalized ladder system, both notions are different since, in a ladder system, given $\delta \in L$, there is exactly one member $A_{\delta}$ of the family with $\sup \left(A_{\delta}\right)=\delta$, while in a generalized ladder system there may be even an uncountable amount of members for which their supremum is $\delta$. The set of all generalized ladder systems in $\omega_{1}$ is easily seen to be inductive respect to set-inclusion, so we may speak of maximal generalized ladder systems.

\section{Lemma 2. A maximal generalized ladder system is a mad family.}

Proof. Let $\mathcal{A}$ be a generalized ladder system which is maximal respect to set-inclusion. Since, from its definition, $\mathcal{A}$ is an almost disjoint family in $\omega_{1}$, we only need showing its maximality. For this, again reasoning by contradiction, let $\mathcal{B}$ 
be an almost disjoint family in $\omega_{1}$ such that $\mathcal{B} \supsetneq \mathcal{A}$. Then, there is $B \in \mathcal{B} \backslash \mathcal{A}$. Since $B$ is an infinite set, we may find a strictly increasing sequence $\left\{b_{j}: \quad j \geq 1\right\} \subseteq B$. Hence, the set $B_{0}:=\left\{b_{j}: j \geq 1\right\}$ satisfies that $B_{0}^{(1)}=\left\{\sup \left(B_{0}\right)\right\}$ and so the collection $\mathcal{A}_{0}:=\mathcal{A} \cup\left\{B_{0}\right\}$ is a generalized ladder system in $\omega_{1}$. But $B_{0} \notin \mathcal{A}$ contradicts the assumption that $\mathcal{A}$ is a maximal generalized ladder system in $\omega_{1}$.

\section{About Monolithic Mrówka Compacta}

A compact $K$ is said to be monolithic whenever each separable subset is second countable, by Uryshon's metrization theorem, this equals to say that each separable subset must be metrizable. The notion of monolithic space is due to Arkhangel'skii, (Arkhangel'skii, A. V., 1992). Translated to scattered compacta, this means that in order to be monolithic every countable subset must have countable closure. One of the best known classes of this type of spaces is the one formed by Corson compacta.

If $X$ is a Banach space such that it has the $C S C P$, it is not hard to see, ( Ferrer, J. et al.,(2013), that the dual unit ball $B_{X^{*}}$ is monolithic respect to the weak-star topology. Consequently, if $C(K)$ has the $C S C P$, since being monolithic is hereditary, it follows that $K$ is monolithic. As we recalled in (Ferrer, J., 2015), under $C H$, a compact $L$ was constructed in (Argyros, S. et al., 1988) such that it is Corson, hence monolithic, but $C(L)$ does not have the $C S C P$, thus proving that $K$ being monolithic is in general not sufficient in order to have that the function space $C(K)$ enjoys the $C S C P$.

The next definition introduces a class of scattered compacta, that we prove strictly contains the monolithic scattered ones, which will later give us a necessary condition for $C\left(K_{\mathcal{A}}\right)$ to have the $S C P$.

Definition 1. If $K$ is a scattered compact, we say that it is almost monolithic whenever the interior of the closure of each countable subset is countable.

Clearly, every monolithic space is almost monolithic, while we see in the next example that the converse is not true, even for Mrówka compacta. We introduce some more notation first, if $\mathcal{A}$ is an almost disjoint family of countably infinite subsets of the infinite set $S$, given a countably infinite subset $N$ of $S$, we define the following subfamily of $\mathcal{A}$

$$
\mathcal{A}^{N}:=\left\{A \in \mathcal{A}: A \subset^{*} N\right\} .
$$

It is easy to prove that

$$
N \cup \mathcal{A}^{N} \subseteq \operatorname{int}(\bar{N}) \subseteq N \cup \mathcal{A}^{N} \cup\{\infty\}
$$

Example. Let

$$
S:=\left[0, w_{1}\left[\backslash \left[0, w_{1}\left[^{(1)},\right.\right.\right.\right.
$$

i.e., $S$ is the set of non-limit countable ordinals. For each $\alpha \in\left[w, w_{1}\left[{ }^{[1)}\right.\right.$, let $M_{\alpha}:=\left\{a_{\alpha, j}: j \geq 1\right\}$ be such that

$$
\forall j, a_{\alpha, j} \in\left[w, w_{1}\left[\backslash \left[w, w_{1}\left[{ }^{(1)}, a_{\alpha, j}<a_{\alpha, j+1}, \sup _{j} a_{\alpha, j}=\alpha .\right.\right.\right.\right.
$$

Let $\mathcal{N}:=\left\{N_{\alpha}: \alpha \in\left[w, w_{1}\left[{ }^{(1)}\right\}\right.\right.$ be an uncountable almost disjoint family of countably infinite subsets of [0, $w$ [. Setting, for each $\alpha \in\left[w, w_{1}\left[{ }^{(1)}\right.\right.$,

$$
A_{\alpha}:=N_{\alpha} \cup M_{\alpha}
$$

we obtain that $\mathcal{A}:=\left\{A_{\alpha}: \alpha \in\left[w, w_{1}\left[{ }^{[1)}\right\}\right.\right.$ is an almost disjoint family in $S$. Let $K_{\mathcal{A}}$ be the associated Mrówka compact. It is easy to see that in order to show that $K_{\mathcal{A}}$ is almost monolithic it suffices to prove that, for each countably infinite subset $L \subseteq S$, the family $\mathcal{A}^{L}$ is countable:

Setting $L_{0}:=L \cap\left[0, w\left[\right.\right.$ and $L_{1}:=L \cap\left[w, w_{1}\left[\backslash\left[w, w_{1}\left[{ }^{[1)}\right.\right.\right.\right.$, we have that $L=L_{0} \cup L_{1}$. Let $\gamma:=\sup (L)<w_{1}$. If $\alpha \in\left[w, w_{1}\left[{ }^{(1)}\right.\right.$ is such that $A_{\alpha} \in \mathcal{A}^{L}$, then $A_{\alpha} \backslash L$ is finite and hence

$$
A_{\alpha} \cap L=\left(A_{\alpha} \cap L_{0}\right) \cup\left(A_{\alpha} \cap L_{1}\right)=\left(N_{\alpha} \cap L_{0}\right) \cup\left(M_{\alpha} \cap L_{1}\right)
$$

is a cofinite subset of $A_{\alpha}$, and so $M_{\alpha} \cap L_{1}$ is an infinite set. Thus, there is an infinite sequence $\left(a_{\alpha, j_{h}}\right)_{h}$ contained in $L_{1}$. Hence $\gamma \geq \sup _{h} a_{\alpha, j_{h}}=\alpha$, i.e., $\mathcal{A}^{L}$ is contained in the family $\left\{A_{\alpha}: \alpha \in\left[w, w_{1}\left[{ }^{(1)} \cap[0, \gamma]\right\}\right.\right.$ which is clearly countable. Thus, after (1), this shows that $\operatorname{int}(\bar{L})$ is countable. We have thus shown that $K_{\mathcal{A}}$ is almost monolithic.

To see that $K_{\mathcal{A}}$ is not monolithic, just notice that the closure of the countable set $[0, w$ [ contains the family $\mathcal{A}$ which is is uncountable.

Let $\mathcal{A}$ be a generalized ladder system in $\omega_{1}$. We say that it has countable type whenever, for each limit ordinal $\delta<\omega_{1}$, the collection

$$
\mathcal{A}_{\delta}:=\left\{A \in \mathcal{A}: A^{(1)}=\{\delta\}\right\}
$$


is a countable one. The following result characterizes the generalized ladder systems whose associated Mrówka compact is monolithic. Notice that the Mrówka compact of the former example is not a generalized ladder system, since, for each $\alpha \in\left[w, w_{1}\left[{ }^{(1)}, A_{\alpha}^{(1)}=\{\omega, \alpha\}\right.\right.$.

Proposition 1. Let $\mathcal{A}$ be a generalized ladder system in $\omega_{1}$ and $K_{\mathcal{A}}$ its associated Mrówka compact. Then the following assertions are equivalent

(i) $K_{\mathcal{A}}$ is almost monolithic.

(ii) $K_{\mathcal{A}}$ is monolithic.

(iii) $\mathcal{A}$ has countable type.

Proof. If $K_{\mathcal{A}}$ is almost monolithic, to see that it is monolithic it suffices to show that, for any countable ordinal $\alpha$, the closure of $\left[0, \alpha\left[\right.\right.$ in $K_{\mathcal{A}}$ is countable. But, as we saw before, this reduces to see that the collection $\mathcal{A}_{[0, \alpha[}$ is a countable one. Thus, if $A \in \mathcal{A}_{[0, \alpha[}$, since $A \cap[0, \alpha[$ is infinite, we have that $\sup (A)=\sup (A \cap[0, \alpha[) \leq \alpha$. Hence, $A \subseteq[0, \alpha]$, that is, with the notation formerly introduced, $A \in \mathcal{A}^{[0, \alpha]}$. We have shown that $\mathcal{A}_{[0, \alpha[} \subseteq \mathcal{A}^{[0, \alpha]}$; since $\mathcal{A}^{[0, \alpha]}$ is contained in int $(\overline{[0, \alpha]})$, which is countable by hypothesis, we have that $\mathcal{A}^{[0, \alpha]}$ is countable and it follows that $\mathcal{A}_{[0, \alpha[}$ is countable too. This proves (i) $\Rightarrow$ (ii).

To show that $(\mathrm{ii}) \Rightarrow(\mathrm{iii})$, if $K_{\mathcal{A}}$ is monolithic, then, for each limit ordinal $\delta<\omega_{1}$, since [0, $\delta$ [ is countable, $\overline{[0, \delta[}$ must be countable. But, using the notation formerly introduced and noticing that

$$
\left[0, \delta\left[\cup \mathcal { A } _ { [ 0 , \delta [ } \subseteq \overline { [ 0 , \delta [ } \subseteq \left[0, \delta\left[\cup \mathcal{A}_{[0, \delta[} \cup\{\infty\}\right.\right.\right.\right.
$$

we have that the collection $\mathcal{A}_{[0, \delta[}$ is countable. Since it is clear that this collection contains $\mathcal{A}_{\delta}$ we have that $\mathcal{A}_{\delta}$ is also countable. It then follows that $\mathcal{A}$ has countable type.

Finally, we see that $(i i i) \Rightarrow(i)$. In order to show that $K_{\mathcal{A}}$ is almost monolithic it suffices to prove that, for each ordinal $\delta<\omega_{1}$, the interior of the closure of $\left[0, \delta\left[\right.\right.$ in $K_{\mathcal{A}}$ is countable. But, as indicated above, this reduces to see that the collection $\mathcal{A}^{[0, \delta[}$ is countable. Now, $A \in \mathcal{A}^{[0, \delta[}$ implies that $A \subset^{*}[0, \delta[$, hence $\sup (A) \leq \delta$, which gives us that

$$
\mathcal{A}^{[0, \delta[} \subseteq\{A \in \mathcal{A}: \sup (A) \leq \delta\}=\left\{A \in \mathcal{A}: A^{(1)}=\{\sup (A)\} \subseteq[0, \delta]\right\}=\bigcup_{\alpha \leq \delta} \mathcal{A}_{\alpha} .
$$

Since this last set is a countable union of countable collections, it follows that it is also countable and so is $\mathcal{A}_{[0, \delta[}$.

\section{A Necessary Condition for the Separable Complementation Property}

Again for the sake of completeness, let us remember that a Banach space $E$ is said to have the separable complementation property whenever each closed separable subspace is contained in a separable complemented subspace. After Sobcyk's theorem, one of the straightforward consequences of this property is that isomorphic copies of $c_{0}$ are always complemented in Banach spaces with the $S C P$, being this one of the main features in the study of this property.

Also seeking self-completeness, let us just say that a Banach space $E$ is said to possess the controlled separable complementation property if, for every two separable subspaces $U$ and $V$ of $E$ and $E^{*}$, respectively, there is a bounded projection $P$ on $E$ such that

(i) $P(E)$ is separable,

(ii) $U \subseteq P(E)$,

(iii) $V \subseteq P^{*}\left(E^{*}\right)$.

Needless saying, the $C S C P$ clearly implies having the $S C P$, while the converse is not true: To see this, as stated in (Banakh, T. et al., 2004), simply consider the space $\ell_{1}\left(\omega_{1}\right)$; as it happens with every space with an unconditional basis, $\ell_{1}\left(\omega_{1}\right)$ has the $S C P$, but, since it is not separable and its dual $\ell_{1}\left(\omega_{1}\right)^{*}=\ell_{\infty}\left(\omega_{1}\right)$ is weak ${ }^{*}$-separable, it follows that $\ell_{1}\left(\omega_{1}\right)$ does not have the CSCP. Also, it is interesting to remark, see (González, A. \& Montesinos, V., 2009), that all weakly Lindelöf determined Banach spaces have the $C S C P$, in particular the weakly compactly generated ones. We say that the Mrówka compact $K_{\mathcal{A}}$ associated to the almost disjoint family $\mathcal{A}$ of countably infinite subsets of the set $S$ is strictly separable whenever $S$ is countable and $\mathcal{A}$ is uncountable.

Proposition 2. If $E$ is a Banach space such that it has the $S C P$, then $E$ contains no isomorphic copies of $C\left(K_{\mathcal{A}}\right)$, where $K_{\mathcal{A}}$ is a strictly separable Mrówka compact.

Proof. Seeking a contradiction, assume that $E$ has the $S C P$ and let $F$ be a closed linear subspace of $E$ such that there is a topological isomorphism $T: C\left(K_{\mathcal{A}}\right) \rightarrow F$, with $K_{\mathcal{A}}$ a strictly separable Mrówka compact. Then, $K_{\mathcal{A}}$ is the Mrówka 
compact associated to the uncountable almost disjoint family $\mathcal{A}$ formed by countably infinite subsets of the countable set $S$. We consider the subspaces

$$
U:=\overline{\operatorname{span}}\left\{1_{s}: s \in S\right\} ; \quad F_{0}:=T(U) .
$$

Since $U$ is isomorphic to $c_{0}$, it is plain that $F_{0}$ is also isomorphic to $c_{0}$.

Making use of Sobcyk's theorem, there is a closed subspace $G$ such that $E=F_{0} \oplus G$. Thus, $F=F_{0} \oplus(G \cap F)$. If $P$ is the projection from $F$ onto $F_{0}$ along $G \cap F$, defining $Q:=T^{-1} P T$, we obtain a bounded linear projection on $C\left(K_{\mathcal{A}}\right)$ such that $Q\left(C\left(K_{\mathcal{A}}\right)\right)=U$. But

$$
U=C_{\mathcal{A} \cup\{\infty\}}\left(K_{\mathcal{A}}\right)
$$

and so

$$
C\left(K_{\mathcal{A}}\right) \simeq U \times C\left(K_{\mathcal{A}}\right) / U \simeq U \times C(\mathcal{A} \cup\{\infty\}) \simeq c_{0} \times c_{0}(\mathcal{A}) .
$$

This implies that $C\left(K_{\mathcal{H}}\right)$ would have to be weakly compactly generated, hence it would have the CSCP. A contradiction since $K_{\mathcal{A}}$ is not monolithic.

In what follows, $X$ will be a Hausdorff locally compact scattered space which is first countable. We give next a couple of definitions in order to achieve a more general necessary condition for $C_{0}(X)$, the space of the continuous functions in $X$ which vanish at infinity, to have the $S C P$. First, notice after (Ferrer, J., 2015) that each point $x$ in $X$ admits a countable clopen neighborhood.

Definition 2. Given a countably infinite subset $A \subseteq X$ and a point $x \in X$, we say that $A$ converges to $x$, which we symbolize as $A \rightarrow x$, whenever, if $U$ is a neighborhood of $x$, then $U \cap A$ is a cofinite subset of $A$.

Let us simply observe that, for a countably infinite set $A, A \rightarrow x$ and $A \rightarrow y$ imply that $x=y$.

Definition 3. A point $x \in X$ is said to have cofinite type whenever there is a countably infinite clopen neighborhood $V$ of $x$ such that the sets of the form $\{x\} \cup A$, where $A$ is a cofinite subset of $V$, are basic neighborhoods of $x$.

Proposition 3. If $C_{0}(X)$ has the $S C P$ then, for each countably infinite open subset $N$ of $X$, either int $(c l(N))$ is countable, or int $(\operatorname{cl}(N)) \backslash N$ contains a point such that it does not have cofinite type.

Proof. Arguing by contradiction, let us assume that there is a countably infinite open subset $N$ of $X$ such that $\operatorname{int}(\operatorname{cl}(N))$ is not countable with all points in $\operatorname{int}(\operatorname{cl}(N)) \backslash N$ having cofinite type. Let us write

$$
\operatorname{int}(\operatorname{cl}(N)) \backslash N=\left\{x_{i}: i \in I\right\},
$$

where $I$ is an uncountable set. For each $i \in I$, since $x_{i}$ has cofinite type, let $V_{i}$ be the countably infinite clopen neighborhood of $x_{i}$, which we may assume that is contained in $\operatorname{int}(\operatorname{cl}(N))$, such that each neighborhood of $x_{i}$ contains a set of the form $\left\{x_{i}\right\} \cup A$, with $A$ being a cofinite subset of $V_{i}$. Clearly, $V_{i} \rightarrow x_{i}$, hence, if $i, j$ are distinct elements of $I$, then $V_{i} \cap V_{j}$ must be a finite set. Besides, $V_{i} \backslash N=\left\{x_{i}\right\}$, otherwise, assuming there is $x \in V_{i} \backslash N \backslash\left\{x_{i}\right\}$, then $x \in \operatorname{int}(\operatorname{cl}(N)) \backslash N$ implies that there is $j \in I, j \neq i$, such that $x=x_{j}$; but this is a contradiction, since then $x_{j} \in V_{i}$ would imply that $V_{i}$, being a neighborhood of $x_{j}$ would contain a cofinite subset of $V_{j}$. Thus, for each $i \in I$, setting $A_{i}:=V_{i} \cap N$, we have that $V_{i}=\left\{x_{i}\right\} \cup A_{i}$. Consequently, we have that the collection $\mathcal{A}:=\left\{A_{i}: i \in I\right\}$ is an uncountable almost disjoint family of countably infinite subsets of the countable set $N$.

Setting $K_{\mathcal{A}}:=N \cup \mathcal{A} \cup\{\infty\}$ to be the associated Mrówka compact, it is clear that $K_{\mathcal{A}}$ is a strictly separable Mrówka compact. We now define the map $\psi: X \rightarrow K_{\mathcal{A}}$ such that, for $x \in X$, we set

$$
\psi(x):= \begin{cases}x, & \text { if } x \in N, \\ A_{i}, & \text { if } x=x_{i}, i \in I, \\ \infty, & \text { elsewhere. }\end{cases}
$$

To see that $\psi$ is continuous, given $x \in X$, let $W$ be a neighborhood of $\psi(x)$ in $K_{\mathcal{A}}$. Then

(a) If $x \in N$, since $N$ is open and $\psi(x)=x$, the set $U:=W \cap N$ is a neighborhood of $x$ in $X$ and clearly $\psi(U)=U \subseteq W$.

(b) If $x=x_{i}$, for some $i \in I$, then $\psi(x)=\psi\left(x_{i}\right)=A_{i}$. Thus, $W$ must contain a set of the form $\left\{A_{i}\right\} \cup B$, where $B$ is a cofinite subset of $A_{i}$. Let $U:=\left\{x_{i}\right\} \cup B$. Then, since $B$ is cofinite in $A_{i}=V_{i} \backslash\left\{x_{i}\right\}$, it follows that $U$ is a neighborhood of $x_{i}$ in $X$. Besides, $\psi(U)=\left\{A_{i}\right\} \cup B \subseteq W$.

(c) If $x \notin N \cup\left\{x_{i}: i \in I\right\}$, then $\psi(x)=\infty$. Hence, $W$ contains a set of the form $K_{\mathcal{A}} \backslash F \backslash A_{i_{1}} \backslash \ldots \backslash A_{i_{n}} \backslash\left\{A_{i_{1}}, \ldots, A_{i_{n}}\right\}$, where $F$ is a finite subset of $N$ and $A_{i_{1}}, \ldots, A_{i_{n}}$ are in $\mathcal{A}$. Taking

$$
U:=X \backslash F \backslash V_{i_{1}} \backslash \ldots \backslash V_{i_{n}},
$$


we have that $U$ is an open set which contains $x$, i.e., a neighborhood of $x$, for which $\psi(U) \subseteq K_{\mathcal{A}} \backslash F \backslash A_{i_{1}} \backslash \ldots \backslash A_{i_{n}} \backslash$ $\left\{A_{i_{1}}, \ldots, A_{i_{n}}\right\} \subseteq W$.

Being $\psi$ clearly onto, we have that the space $C\left(K_{\mathcal{A}}\right)$ is isometric to a subspace of $C_{0}(X)$. Now, since $K_{\mathcal{A}}$ is a strictly separable Mrówka compact, after Proposition 2, we conclude that $C_{0}(X)$ cannot have the $S C P$.

Noticing that in a Mrówka compact $K_{\mathcal{A}}$ each point in $K_{\mathcal{A}} \backslash\{\infty\}$ has cofinite type and that $C_{0}\left(K_{\mathcal{A}} \backslash\{\infty\}\right)$ is isomorphic to $C_{\infty}\left(K_{\mathcal{A}}\right)$, the next result obtains.

Corollary 1. If $C\left(K_{\mathcal{A}}\right)$ has the $S C P$, then $K_{\mathcal{A}}$ is almost monolithic.

To see that the converse of the above corollary does not hold, we consider the Mrówka compact constructed in the example given before: $K_{\mathcal{A}}=S \cup \mathcal{A} \cup\{\infty\}$, with $S=\left[0, w\left[\cup\left(\left[w, w_{1}\left[\backslash\left[w, w_{1}\left[{ }^{(1)}\right)\right.\right.\right.\right.\right.\right.$ and $\mathcal{A}:=\left\{A_{\alpha}: \alpha \in\left[w, w_{1}\left[{ }^{(1)}\right\}\right.\right.$, where $A_{\alpha}:=N_{\alpha} \cup M_{\alpha}, \alpha \in\left[w, w_{1}\left[{ }^{(1)}\right.\right.$, being $\mathcal{N}=\left\{N_{\alpha}: \alpha \in\left[w, w_{1}\left[{ }^{(1)}\right\}\right.\right.$ a mad family in $\left[0, \omega\left[\right.\right.$ and $\left\{M_{\alpha}: \alpha \in\left[w, w_{1}\left[{ }^{(1)}\right\}\right.\right.$ a ladder system in $\left[w, w_{1}\left[\backslash\left[w, w_{1}\left[^{(1)}\right.\right.\right.\right.$.

We show that $C\left(K_{\mathcal{A}}\right)$ does not have the $S C P$. For the sake of commodity, let $E:=C\left(K_{\mathcal{A}}\right)$ and we consider the closed linear subspace $F:=\overline{\operatorname{span}}\left\{1_{n}: n<w\right\}$. If $E$ has the $S C P$, then, using Sobcyk's theorem, $F$ is complemented in $E$. But, since $F=\left\{f \in E: f_{\mid\left(\left[w, w_{1}\left[\backslash\left[w, w_{1}[(1) \cup \mathcal{A} \cup\{\infty\}\right.\right.\right.\right.}=0\right\}$, we have

$$
E \simeq F \times E / F \simeq F \times C\left(\left(\left[w, w_{1}\left[\backslash\left[w, w_{1}\left[{ }^{(1)}\right) \cup \mathcal{A} \cup\{\infty\}\right) \simeq c_{0} \times C\left(K_{\mathcal{A}_{0}}\right)\right.\right.\right.\right.
$$

where $K_{\mathcal{A}_{0}}$ is the Mrówka compact associated to $S_{0}:=\left[w, w_{1}\left[\backslash\left[w, w_{1}\left[{ }^{(1)}\right.\right.\right.\right.$ and $\mathcal{A}_{0}:=\left\{A_{\alpha} \cap S_{0}: \alpha \in\left[w, w_{1}\left[{ }^{(1)}\right\}=\left\{M_{\alpha}:\right.\right.\right.$ $\alpha \in\left[w, w_{1}\left[{ }^{(1)}\right\}\right.$. We know that $C\left(K_{\mathcal{F}_{0}}\right)$ has the $C S C P$ (given that $K_{\mathcal{A}_{0}}$ is really a ladder system in $S_{0}$, and we know that ladder systems are always monolithic), hence we have that $E=C\left(K_{\mathcal{A}}\right)$ would enjoy the $C S C P$, a contradiction since the space $K_{\mathcal{A}}$ is not monolithic.

After Proposition 1 and the previous corollary, the following result is straightforward.

Corollary 2. Let $\mathcal{A}$ be a generalized ladder system. The following assertions are equivalent:

(i) $C\left(K_{\mathcal{A}}\right)$ has the $S C P$.

(ii) $K_{\mathcal{A}}$ is almost monolithic.

(iii) $\mathcal{A}$ has countable type.

(iv) $K_{\mathcal{A}}$ is monolithic.

(v) $C\left(K_{\mathcal{A}}\right)$ has the $C S C P$

Since mad families produce Mrówka compacta which are never monolithic, from Lemma 2 and the previous corollary the following result follows.

Corollary 3. Let $\mathcal{A}$ be a maximal generalized ladder system in $\omega_{1}$. Then $C\left(K_{\mathcal{A}}\right)$ does not have the SCP.

Given that, if $\mathcal{A}$ is a mad family in $\omega_{1}$, its associated Mrówka compact $K_{\mathcal{A}}$ is not monolithic, it is clear that $C\left(K_{\mathcal{A}}\right)$ does not have the CSCP. Nevertheless, concerning the $S C P$, although we have just seen in the previous corollary that there are mad families for which their space of continuous functions on the associated Mrówka compact does not enjoy the $S C P$, it is still unknown for us wether in general, for an arbitrary mad family, such a space may still have the $S C P$. Hence we formulate the following related questions.

Question 1. If $\mathcal{A}$ is any mad family in $\omega_{1}$, can $C\left(K_{\mathcal{A}}\right)$ have the $S C P$ ?

A positive answer to the next set-combinatoric question would yield a negative answer to the former one.

Question 2. If $\mathcal{A}$ is any mad family in $\omega_{1}$, does there exist $\delta<\omega_{1}$ for which $\mathcal{A}^{[0, \delta[}$ is uncountable ?

\section{References}

Argyros, S. Mercourakis, S., \& Negrepontis, S. (1988). Functional analytic properties of Corson-compact spaces. Studia Math. 89, 197-229.

Arkhangel'skii, A. V. (1992). Topological Function Spaces, Kluwer.

Avilés, A., \& Moreno, Y. (2008). Automorphisms in spaces of continuous functions on Valdivia compacta. Topology Appl.,155, 2027-2030. 
Banakh, T., Plichko, A., \& Zagorodnyuk, A. (2004). Zeros of quadratic functionals on nonseparable spaces. Colloq. Math., 100, 141-147. https://doi.org/10.4064/cm100-1-13

Dow, A., \& Vaughan, J. E. (2009). Mrówka maximal almost disjoint families for uncountable cardinals. Topology Appl., https://doi.org/10.1016/j.topol..08.024.

Ferrer, J. (2009). On the Controlled Separable Projection Property for some $C(K)$ spaces. Acta Math. Hung. 124, 1-2, 145-154. https://doi.org/10.1007/s10474-009-8165-3

Ferrer, J., \& Wójtowicz, M. (2011). The Controlled Separable Projection Property for Banach spaces. Cent. Eur. J. Math. 9, 1252-1266. https://doi.org/10.2478/s11533-011-0096-х

Ferrer, J., Koszmider, P., \& Kubiś, W. (2013). Almost disjoint families of countable sets and separable complementation properties. J. Math. Anal. Appl., 401, 939-949.

Ferrer, J. (2014). The Controlled Separable Complementation Property and mononolithic compacta. Banach J. Math. Anal., 8(2), 67-78. https://doi.org/10.15352/bjma/1396640052

Ferrer, J. (2015). A note on mononolithic scattered compacta, J. Math. Anal. Appl., 421, 950-954. https://doi.org/10.1016/j.jmaa.2014.07.011

González, A., \& Montesinos, V. (2009). A note on weakly Lindelöf determined Banach spaces. Czechoslovak Math. J., 59(134), 613-621. https://doi.org/10.1007/s10587-009-0055-x

Jech, T. (2003). Set Theory, The third millenium edition, in: Springer Monographs in Mathematics. Springer-Verlag, Berlin.

Mrówka, S. (1977). Some set-theoretic constructions in topology£ Fund. Math., 94, 83-92.

Pol, R. (1979). A function space $C(K)$ which is weakly Lindelöf but not weakly compactly generated, Studia Math. 64 (3) , 279-285.

\section{Copyrights}

Copyright for this article is retained by the author(s), with first publication rights granted to the journal.

This is an open-access article distributed under the terms and conditions of the Creative Commons Attribution license (http://creativecommons.org/licenses/by/4.0/). 